\title{
Consensus guidelines for postoperative stereotactic body radiation therapy for spinal metastases: results of an international survey
}

\author{
Kristin J. Redmond, MD, MPH, ${ }^{1}$ Simon S. Lo, MD, ${ }^{2}$ Scott G. Soltys, MD, ${ }^{3}$ Yoshiya Yamada, MD, ${ }^{4}$ \\ Igor J. Barani, MD, ${ }^{5}$ Paul D. Brown, MD, ${ }^{6}$ Eric L. Chang, MD, ${ }^{7}$ Peter C. Gerszten, MD, ${ }^{8}$ \\ Samuel T. Chao, MD, ${ }^{9}$ Robert J. Amdur, MD, ${ }^{10}$ Antonio A. F. De Salles, MD, PhD, ${ }^{11}$ \\ Matthias Guckenberger, MD, ${ }^{12}$ Bin S. Teh, MD, ${ }^{13}$ Jason Sheehan, MD, PhD, ${ }^{14}$ Charles R. Kersh, MD, ${ }^{15}$ \\ Michael G. Fehlings, MD, PhD, FRCSC, ${ }^{16}$ Moon-Jun Sohn, MD, PhD, ${ }^{17}$ Ung-Kyu Chang, MD, ${ }^{18}$ \\ Samuel Ryu, MD, ${ }^{19}$ Iris C. Gibbs, MD, ${ }^{3}$ and Arjun Sahgal, MD, FRCPC ${ }^{20}$
}

1Department of Radiation Oncology and Molecular Radiation Sciences, The John Hopkins University, Baltimore, Maryland; 2Department of Radiation Oncology, University Hospitals Seidman Cancer Center, Case Comprehensive Cancer Center; ${ }^{9}$ Department of Radiation Oncology, Rose Ella Burkhardt Brain Tumor and Neuro-oncology Center, Cleveland Clinic, Cleveland, Ohio; ${ }^{3}$ Department of Radiation Oncology, Stanford Cancer Institute, Stanford University, Stanford; 5Department of Radiation Oncology, University of California, San Francisco; ' Department of Radiation Oncology, Norris Cancer Center and Keck School of Medicine at University of Southern California; ${ }^{11}$ Department of Neurological Surgery, Brain Research Institute, University of California, Los Angeles, California; ${ }^{4}$ Department of Radiation Oncology, Memorial Sloan Kettering Cancer Center, New York; ${ }^{19}$ Department of Radiation Oncology, Stony Brook Cancer Center, Stony Brook, New York; ${ }^{6}$ Department of Radiation Oncology, MD Anderson Cancer Center; ${ }^{13}$ Department of Radiation Oncology, Houston Methodist Hospital, Cancer Center and Research Institute, Weill Cornell Medical College, Houston, Texas; ${ }^{8}$ Department of Neurological Surgery and Radiation Oncology, University of Pittsburgh Medical Center, Pittsburgh, Pennsylvania; ${ }^{10}$ Department of Radiation Oncology, University of Florida College of Medicine, Gainesville, Florida; ${ }^{12}$ Department of Radiation Oncology, University of Zurich, Switzerland; ${ }^{14}$ Department of Neurological Surgery, University of Virginia Health System, Charlottesville; ${ }^{15}$ Department of Radiation Oncology, Riverside Radiation Oncology Specialists, Newport News, Virginia; ${ }^{16}$ Division of Neurosurgery and Spine Program, Toronto Western Hospital, University of Toronto; ${ }^{20}$ Department of Radiation Oncology, Sunnybrook Health Sciences Center, University of Toronto, Toronto, Ontario, Canada; ${ }^{17}$ Department of Neurological Surgery, Radiosurgery Center, Inje University Ilsan Paik Hospital, College of Medicine, Goyang; and ${ }^{18}$ Department of Neurosurgery, Korea Institute of Radiological and Medical Sciences, Seoul, South Korea

OBJECTIVE Although postoperative stereotactic body radiation therapy (SBRT) for spinal metastases is increasingly performed, few guidelines exist for this application. The purpose of this study is to develop consensus guidelines to promote safe and effective treatment for patients with spinal metastases.

METHODS Fifteen radiation oncologists and 5 neurosurgeons, representing 19 centers in 4 countries and having a collective experience of more than 1300 postoperative spine SBRT cases, completed a 19-question survey about postoperative spine SBRT practice. Responses were defined as follows: 1) consensus: selected by $\geq 75 \%$ of respondents; 2 ) predominant: selected by $50 \%$ of respondents or more; and 3) controversial: no single response selected by a majority of respondents.

RESULTS Consensus treatment indications included: radioresistant primary, 1-2 levels of adjacent disease, and previous radiation therapy. Contraindications included: involvement of more than 3 contiguous vertebral bodies, ASIA Grade A status (complete spinal cord injury without preservation of motor or sensory function), and postoperative Bilsky Grade 3 residual (cord compression without any CSF around the cord). For treatment planning, co-registration of the preoperative MRI and postoperative T1-weighted MRI (with or without gadolinium) and delineation of the cord on the T2-weighted MRI (and/or CT myelogram in cases of significant hardware artifact) were predominant. Consensus GTV (gross tumor

ABBREVIATIONS ASIA = American Spinal Injury Association; CTV = clinical tumor volume; GTV = gross tumor volume; MESCC = malignant epidural spinal cord compression; PRV = planning risk volume; PTV = planning target volume; RT = radiation therapy; RTOG = Radiation Therapy Oncology Group; SBRT = stereotactic body radiation therapy.

SUBMITTED January 22, 2016. ACCEPTED August 5, 2016.

INCLUDE WHEN CITING Published online November 11, 2016; DOI: 10.3171/2016.8.SPINE16121. 
volume) was the postoperative residual tumor based on MRI. Predominant CTV (clinical tumor volume) practice was to include the postoperative bed defined as the entire extent of preoperative tumor, the relevant anatomical compartment and any residual disease. Consensus was achieved with respect to not including the surgical hardware and incision in the CTV. PTV (planning tumor volume) expansion was controversial, ranging from 0 to $2 \mathrm{~mm}$. The spinal cord avoidance structure was predominantly the true cord. Circumferential treatment of the epidural space and margin for paraspinal extension was controversial. Prescription doses and spinal cord tolerances based on clinical scenario, neurological compromise, and prior overlapping treatments were controversial, but reasonable ranges are presented. Fifty percent of those surveyed practiced an integrated boost to areas of residual tumor and density override for hardware within the beam path. Acceptable PTV coverage was controversial, but consensus was achieved with respect to compromising coverage to meet cord constraint and fractionation to improve coverage while meeting cord constraint.

CONCLUSIONS The consensus by spinal radiosurgery experts suggests that postoperative SBRT is indicated for radioresistant primary lesions, disease confined to 1-2 vertebral levels, and/or prior overlapping radiotherapy. The GTV is the postoperative residual tumor, and the CTV is the postoperative bed defined as the entire extent of preoperative tumor and anatomical compartment plus residual disease. Hardware and scar do not need to be included in CTV. While predominant agreement was reached about treatment planning and definition of organs at risk, future investigation will be critical in better understanding areas of controversy, including whether circumferential treatment of the epidural space is necessary, management of paraspinal extension, and the optimal dose fractionation schedules.

https://thejns.org/doi/abs/10.3171/2016.8.SPINE16121

KEY WORDS postoperative spine stereotactic body radiation therapy; SBRT; spinal metastases; oncology; consensus guidelines

$\mathrm{E}$ VIDENCE of spinal metastases may be found in as many as $90 \%$ of cancer patients ${ }^{20,36}$ upon autopsy, and $40 \%$ of patients with metastatic disease will present with clinically evident spinal metastases. ${ }^{18}$ Treatment options for patients with spinal metastases are dependent on individual patient circumstances, but include systemic therapy, radiation therapy (RT), surgery, and surgery plus RT.

The role of surgery for patients with malignant epidural spinal cord compression (MESCC) was clarified by a randomized controlled study comparing the efficacy of $\mathrm{RT}$ alone to decompressive surgery plus RT. The RT was administered using conventional 3D treatment planning to a dose of 30 Gy in 10 fractions. Patients in the surgical arm had significant benefits with respect to the duration of ambulatory function, decreased use of corticosteroids and opioid analgesics, and longer overall survival. As a result, in a highly select group of patients with a single area of symptomatic MESCC ${ }^{25}$ decompressive surgery followed by RT is considered the standard of care. More recently, combined surgery and RT was shown to substantially enhance quality of life in a prospective, multicenter study. ${ }^{8}$ Surgery is also considered in patients with mechanical instability. Several researchers have attempted to classify degrees of instability. Most recently, the Spinal Instability Neoplastic Score (SINS) ${ }^{9}$ has emerged as a validated scale of instability to guide surgical decision making.

The goals of surgery are tumor debulking, neural decompression, and spine stability, but surgery alone is not typically suited to achieve durable local control. Rates of local recurrence with surgery and heterogeneous adjuvant therapy, including radiation, chemotherapy, and/or hormone therapy, are as high as $70 \%$ at 1 year and $96 \%$ at 4 years. ${ }^{17}$ To reduce this risk, patients have historically been treated with conventional RT, often over a 2- to 3-week period, and radiation following surgery is considered the standard of care. When standard 3D techniques are used, the spinal canal receives the full prescription radiation dose, which must be safe with respect to spinal cord injury and, hence, not necessarily efficacious with respect to maximizing local tumor control. As a result, local recurrence rates following conventional RT remain as high as $34 \%$, $7,8,13,15,25,28$

Over the past decade, significant advances have been made to allow precise delivery of RT using stereotactic techniques with submillimeter accuracy. Stereotactic body radiation therapy (SBRT) allows safe delivery of higher biologically equivalent doses in fewer fractions. Data from retrospective and prospective studies suggest that spinal SBRT for intact vertebral metastases is associated with high rates of local control with a low risk of marginal failure even in radioresistant histologies. . $^{1,511,16,27,30}$ NRG Oncology/RTOG (Radiation Therapy Oncology Group) 0631 ("Image-Guided Radiosurgery or Stereotactic Body Radiation Therapy in Treating Patients With Localized Spine Metastasis," clinicaltrials.gov identifier NCT00922974) is an ongoing randomized controlled trial comparing spinal SBRT with conventional radiation therapy. Formal consensus guidelines have been developed to guide the treatment of intact vertebral bodies. ${ }^{6}$

The practice of spinal SBRT in postoperative patients is similarly growing, and data are emerging to suggest that outcomes with this technique may be superior to those achieved with conventional RT., 2,3,12,19,22,24,27,33,34 In contrast to the situation with spine SBRT for intact vertebral metastases, however, there are presently few recommendations to guide the use of SBRT for spinal metastases after spinal surgery. The approach is technically challenging, and there are numerous considerations unique to the postoperative spine patient. The purpose of this study was to develop international consensus guidelines to help guide safe practice in this emerging field. 


\section{Methods}

Twenty spine oncology specialists representing 19 centers in the US, Canada, Germany, and Korea completed a 19-question survey about postoperative spine SBRT practice. Participants were selected based on prior publications in spine SBRT as well as the volume of postoperative spine SBRT patient treated in clinical practice. Questions were asked regarding the following topics: indications and contraindications of postoperative spine SBRT; grade of postoperative residual epidural disease that is appropriate for SBRT; image selection and fusion into radiation treatment planning systems; gross tumor volume (GTV), clinical target volume (CTV), planning target volume (PTV) delineation; cord avoidance structure and whether it should be subtracted from PTV; situations warranting circumferential treatment of the epidural space; management of paraspinal extension; dose and fractionation schedules in various clinical scenarios as manually entered by respondents into a table; spinal cord constraint in various treatment scenarios; accounting for repair in selecting a spinal cord constraint in the retreatment setting; use of an integrated boost to areas of gross disease within the CTV; accounting for dose perturbation and artifact in the setting of spinal instrumentation; use of RTOG-approved treatment planning algorithms; acceptable PTV coverage; and the approach if unable to meet the target PTV coverage while meeting normal tissue constraints. The full survey is available online in the Appendix.

Responses were defined as follows: 1) consensus: selected by at least $75 \%$ of respondents; 2) predominant: selected by at least $50 \%$ of respondents; 3) controversial: no single response selected by a majority of respondents. Descriptive statistics were used to review results.

\section{Results}

Fifteen radiation oncologists and five neurosurgeons with a collective experience of more than 1300 postoperative spine SBRT cases (range 20 to more than 100 cases) completed the survey. The mean time in practice for the providers who completed the survey was 14.7 years (range 5-30 years). The consensus indications and contraindications for postoperative spine SBRT are outlined in Table 1.

\section{Image Fusion, Target Volume, and Organ at Risk Delineation}

For target volume delineation, the predominance of providers co-register the preoperative MRI and postoperative T1-weighted MRI (obtained with and/or without gadolinium) to the CT simulation. In addition, for spinal cord delineation either T2-weighted MRI or a CT myelogram, in cases of significant hardware artifact, is similarly co-registered.

The consensus GTV is the postoperative residual tumor based on MRI. The predominance of providers delineate the CTV as the postoperative tumor bed (defined as the entire extent of preoperative tumor), relevant anatomical compartment, and any residual disease. With reconstitution of the thecal sac, the CTV margin is the thecal sac rather than the previously compressed dura, as tumor rarely violates this barrier. The consensus is that the surgical
TABLE 1. Consensus indications and contraindications for postoperative spine SBRT

\begin{tabular}{cc}
\hline \multicolumn{1}{c}{ Indications } & Contraindications \\
\hline $\begin{array}{l}\text { Radio-resistant primary } \\
\text { 1-2 levels of adjacent } \\
\text { disease }\end{array}$ & $\begin{array}{c}\text { Involvement of more than 3 contiguous } \\
\text { vertebral bodies } \\
\text { Prior overlapping radia- } \\
\text { tion therapy }\end{array}$ \\
& ASIA Grade A status (complete spinal cord \\
& injury without preservation of motor or \\
& sensory function) \\
& Postoperative Bilsky Grade 3 residual \\
& (spinal cord compression without any \\
& CSF around the spinal cord) \\
\hline
\end{tabular}

instrumentation and incision do not need to be included in the CTV unless believed to be specifically at risk due to the surgical approach or direct involvement by tumor. Circumferential treatment of the epidural space as depicted in Fig. 1 was controversial. The PTV expansion ranges from 0 to $2 \mathrm{~mm}$, and the margin for paraspinal extension was controversial, ranging from no additional expansion up to a 5-mm expansion in these regions.

The consensus spinal cord is the true cord based on either T2-weighted MRI or the CT myelogram. For the spinal cord planning risk volume (PRV), the predominance of providers use the true spinal cord, although the practice varies and many use a 1.5-2 mm expansion from the true cord or the thecal sac without expansion. The predominance of providers subtract the spinal cord PRV from their PTV expansion.

Table 2 outlines delineation of the target volume and spinal cord avoidance structures for postoperative spine SBRT, and Fig. 2 shows a sample treatment plan for a patient with an L-1 metastasis who has undergone surgical decompression and stabilization.

\section{Dose/Fractionation Schedules, Spinal Cord Constraints, and Target Volume Coverage}

Prescription doses and fractionation schedules were controversial. For re-irradiation following a previous course of irradiation, providers predominantly account for repair and the time interval between prior RT and spinal SBRT in calculating cord constraints. Common dose and fractionation schemes for various clinical situations are presented in Table 3.

Exactly half of the providers who completed the survey use an integrated boost to areas of residual tumor. For patients with radiosensitive tumors, sample integrated boost doses to the GTV were 16-22 Gy in a single fraction. For patients with radioresistant tumors, sample integrated boost doses to the GTV were $18-25$ Gy in a single fraction or $50 \mathrm{~Gy}$ in 5 fractions. In addition, exactly half of the providers use a density override for hardware within the beam path. The consensus was that a treatment planning algorithm approved by the RTOG for calculation of dose within a medium with heterogeneities should be used for all postoperative spine SBRT cases. Example algorithms are shown in Table 4.

Spinal cord constraints for the cord PRV were also controversial, but common schemes according to the fractionation schedule and prior radiation doses are provided in 


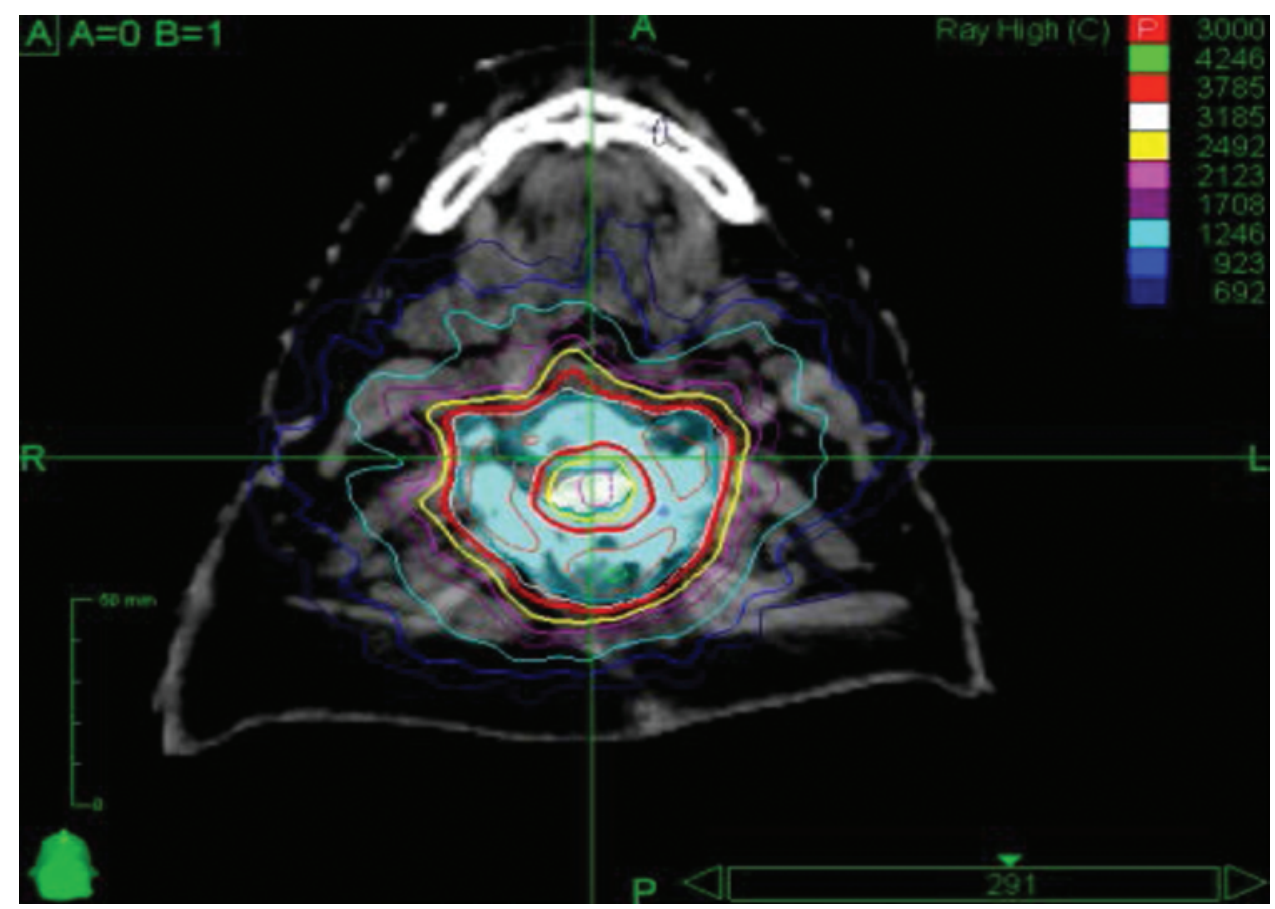

FIG. 1. Example of a circumferential treatment plan for a patient treated with $3000 \mathrm{cGy}$ in 5 fractions. Circumferential treatment of the epidural space is controversial as it is challenging from the treatment planning perspective and generally requires greater compromise of the epidural space to meet spinal cord constraints. Nonetheless, the most common practice is to treat circumferentially in patients with circumferential disease preoperatively, although some providers do this only when there is gross residual circumferential disease postoperatively, while others avoid it in all patients. Republished with permission of Elsevier Science and Technology Journals, from Postoperative stereotactic body radiation therapy (SBRT) for spine metastases: a critical review to guide practice, Redmond et al., Int J Radiat Oncol Biol Phys 95(5):1414-1428, 2016; permission conveyed through Copyright Clearance Center, Inc. Figure is available in color online only.

Table 5. Accounting for the time between initial treatment and retreatment in determining spinal cord constraints is controversial. While there is no common algorithm to account for repair, the predominance of providers use more conservative spinal cord constraints in previously irradiated patients. Acceptable PTV coverage is controversial. Regarding dosimetric target, most of the respondents indicated that their preference was for $90 \%$ of the prescription dose to cover $90 \%$ of the PTV, but the majority of those who indicated a different preference aimed to have
$95 \%-100 \%$ of the prescription dose cover $95 \%$ of the PTV. The predominance of participating providers indicated that they will compromise PTV coverage to meet spinal cord constraints and/or fractionate the prescription dose further to improve coverage while meeting the spinal cord constraint.

\section{Discussion}

The standard of care in patients with good performance

TABLE 2. Consensus and predominant practices for GTV, CTV, PTV, spinal cord, and spinal cord PRV delineation for postoperative spine SBRT

\begin{tabular}{ll}
\hline \multicolumn{1}{c}{ Volume } & \multicolumn{1}{c}{ Include } \\
\hline Gross tumor volume (GTV) & Postoperative residual based on MRI \\
\hline Clinical tumor volume (CTV) & Entire extent of preoperative tumor, anatomic compartment involved, \& any postoperative residual \\
& Surgical instrumentation \& incision not included unless involved \\
& Prophylactic circumferential treatment of epidural space controversial \\
& Additional expansion up to $5 \mathrm{~mm}$ for paraspinal extension controversial \\
& Consider an additional expansion of up to $5 \mathrm{~mm}$ cranio-caudally beyond known epidural disease \\
& extent based on pre- \& postoperative imaging \\
\hline Planning target volume (PTV) & 0- to 2-mm expansion from CTV \\
\hline Spinal cord & True spinal cord based on postoperative T2-weighted MRI or CT myelogram in cases of significant \\
& hardware artifact \\
\hline Spinal cord planning risk volume (PRV) & 0- to 2-mm expansion of spinal cord volume \\
\hline
\end{tabular}



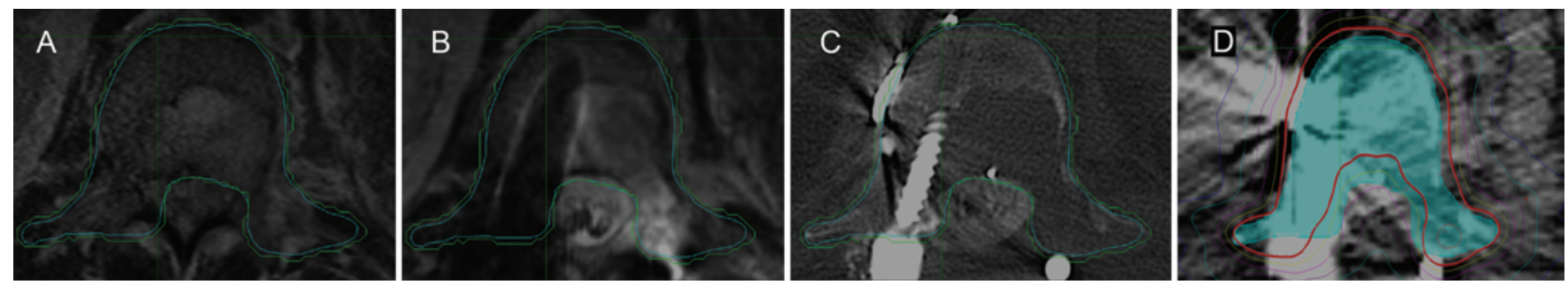

FIG. 2. Sample treatment plan for a patient with metastatic renal cell carcinoma and an L-1 metastasis who had undergone surgical decompression and stabilization. A: Preoperative T1-weighted post-gadolinium MR image showing tumor involvement of the vertebral body and pedicles and extension into the epidural space with tumor encompassing the cauda equina. B and C: Postoperative T2-weighted MR image (B) and CT myelogram (C) showing decompression of the thecal sac and placement of surgical instrumentation for stabilization. D: Final postoperative spine SBRT treatment plan. The red line represents the prescription isodose line. The blue contour is the thecal sac without margin which serves as the cord avoidance structure. It is subtracted out from the PTV (shown as the green contour) to meet the spinal cord constraints. Republished with modification (reformatted) with permission of Elsevier Science and Technology Journals, from Postoperative stereotactic body radiation therapy (SBRT) for spine metastases: a critical review to guide practice, Redmond et al., Int J Radiat Oncol Biol Phys 95(5):1414-1428, 2016; permission conveyed through Copyright Clearance Center, Inc. Figure is available in color online only.

status, oligo-metastatic disease, and vertebral metastases causing instability or MESCC with neurological deficits is surgical decompression and/or stabilization followed by RT. Historically, conventional radiation techniques using simple parallel opposed anteroposterior/posteroanterior treatment fields have been used. Unfortunately, because all normal structures within the beam's path receive the full dose, the prescription must be limited to respect the radiation tolerance of the spinal cord. As a result, the rate of local recurrence is high. With improvements in systemic therapy, including targeted agents and immunotherapy, survivorship in patients with metastatic cancer is increasing, and as a result, durable local control following surgical management of spine metastases has become critical. Management of recurrent disease in a previously treated vertebra is also challenging and associated with a high risk of permanent neurological sequelae, resulting in both a significant degradation in patients' quality of life as well as an exponential increase in healthcare costs.

TABLE 3. Common dose and fractionation schemes based on clinical scenario and prior overlapping RT doses

\begin{tabular}{|c|c|}
\hline Clinical Scenario & $\begin{array}{c}\text { Reasonable Dose/ } \\
\text { Fractionation Schedules }\end{array}$ \\
\hline $\begin{array}{l}\text { No prior RT, single vertebral level, } \\
\text { no epidural disease }\end{array}$ & $\begin{array}{l}16-24 \mathrm{~Gy} \times 1 \text { fraction } \\
12 \mathrm{~Gy} \times 2 \text { fractions } \\
8-10 \mathrm{~Gy} \times 3 \text { fractions }\end{array}$ \\
\hline $\begin{array}{l}\text { No prior RT, multiple vertebral } \\
\text { levels \&/or epidural disease }\end{array}$ & $\begin{array}{l}16-18 \mathrm{~Gy} \times 1 \text { fraction } \\
12 \mathrm{~Gy} \times 2 \text { fractions } \\
8-10 \mathrm{~Gy} \times 3 \text { fraction } \\
6-8 \mathrm{~Gy} \times 5 \text { fractions }\end{array}$ \\
\hline No prior RT, epidural disease & $\begin{array}{l}16-18 \mathrm{~Gy} \times 1 \text { fraction } \\
12 \mathrm{~Gy} \times 2 \text { fractions } \\
8 \mathrm{~Gy} \times 4 \text { fractions } \\
6-7 \mathrm{~Gy} \times 5 \text { fractions }\end{array}$ \\
\hline $\begin{array}{l}\text { Prior RT, any extent of epidural } \\
\text { disease }\end{array}$ & $\begin{array}{l}16-18 \mathrm{~Gy} \times 1 \text { fraction } \\
12 \mathrm{~Gy} \times 2 \text { fractions } \\
7-10 \mathrm{~Gy} \times 3 \text { fractions } \\
8-10 \mathrm{~Gy} \times 4 \text { fractions } \\
5-7 \mathrm{~Gy} \times 5 \text { fractions }\end{array}$ \\
\hline
\end{tabular}

Over the past decade, advances in technology, including on-board image guidance, micro-multileaf collimation, robotic technology, and highly reproducible immobilization devices, have allowed the emergence of SBRT techniques that deliver RT with submillimeter accuracy. As a result, it is now possible to use ablative high biologically equivalent doses to tumors in the spinal column and paravertebral space while respecting the tolerance of the immediately adjacent critical normal tissues. Numerous studies have demonstrated excellent rates of local control in patients with metastases in intact vertebral bodies. ${ }^{1,5,11,16,27,30}$ The practice has become the standard of care at many institutions for patients with oligometastatic disease, and consensus contouring guidelines have been published. ${ }^{6}$ Emerging data suggest comparable efficacy in the postoperative setting, 2,3,12,19,22,24,27,33,34 and the practice has been increasingly used in both academic and private practice settings. Nonetheless, few guidelines exist to promote safe and effective application of this emerging technology.

This survey of an international group of spine oncology specialists was designed to develop guidelines to promote safe and effective practice. While high-quality prospective

TABLE 4. Treatment planning algorithms approved by legacy RTOG for calculation of dose within a medium with heterogeneities

\begin{tabular}{ll}
\hline Treatment Planning System & \multicolumn{1}{c}{ Algorithm } \\
\hline BrainLab & Monte Carlo \\
\hline Corvus & Monte Carlo \\
\hline CyberKnife Multiplan & Monte Carlo \\
\hline Eclipse & AAA \\
\hline Eclipse & Acuros \\
\hline Helax & Collapsed cone \\
\hline In house & Monte Carlo \\
\hline Monaco & Monte Carlo \\
\hline Pinnacle & Collapsed cone convolution-adaptive \\
& convolve \\
\hline Tomotherapy & Convolution superposition \\
\hline XiO & Superposition-fast superposition \\
\hline
\end{tabular}

AAA = analytical anisotropic algorithm . 
TABLE 5. Common spinal cord constraints that are applied to either true cord or a surrogate of the true cord (cord PRV or thecal sac) according to no prior and common prior radiation dose exposure

\begin{tabular}{|c|c|c|c|c|c|}
\hline Prior Conventional RT dose & 1 Fraction & 2 Fractions & 3 Fractions & 4 Fractions & 5 Fractions \\
\hline No prior RT \& no cord compromise & $\begin{array}{l}10-14 \text { Gy } D_{\max } \\
10 \text { Gy to }<10 \% \text { cord* }\end{array}$ & 17 Gy $D_{\max }$ & $18-21$ Gy $D_{\max }$ & $23-26$ Gy $D_{\max }$ & $25-30$ Gy $D_{\max }$ \\
\hline No prior RT but cord compromise & $\begin{array}{l}8-14 \text { Gy } D_{\max } \\
10 \text { Gy to }<10 \% \text { cord* }\end{array}$ & 17 Gy $D_{\max }$ & $18-21$ Gy $D_{\max }$ & $23-26$ Gy $D_{\max }$ & $25-28$ Gy $D_{\max }$ \\
\hline 800 cGy in 1 fraction & $9 \mathrm{~Gy}_{\max }$ & $12.2 \mathrm{~Gy} \mathrm{D}_{\max }$ & $14-21 G y D_{\max }$ & $16.2 G_{y} D_{\max }$ & $17.5-27.5 D_{\max }$ \\
\hline 2000 cGy in 5 fractions & 9-12 Gy $D_{\max }$ & $12.2 \mathrm{~Gy} \mathrm{D}_{\max }$ & $14-21 G y D_{\max }$ & $16.2 \mathrm{~Gy} \mathrm{D}_{\max }$ & $15-27.5$ Gy $D_{\max }$ \\
\hline 3000 cGy in 10 fractions & 9-12 Gy $D_{\max }$ & $12.2 \mathrm{~Gy} \mathrm{D}_{\max }$ & $14-21 G y D_{\max }$ & $16.2 \mathrm{~Gy}-24 \mathrm{~Gy} \mathrm{D}_{\max }$ & $17.5-26 G_{y} D_{\max }$ \\
\hline 4000 cGy in 20 fractions & $9-12$ Gy $D_{\max }$ & $12.2 \mathrm{~Gy} \mathrm{D}_{\max }$ & $14-21 G y D_{\max }$ & $16.2 \mathrm{~Gy} \mathrm{D}_{\max }$ & $12-25 G y D_{\max }$ \\
\hline $4500 \mathrm{cGy}$ in 25 fractions & 9-12 Gy $D_{\max }$ & $12.2 \mathrm{~Gy} \mathrm{D}_{\max }$ & $14-21$ Gy $D_{\max }$ & $16.2 \mathrm{~Gy}_{\max }$ & $12-18$ Gy $D_{\max }$ \\
\hline
\end{tabular}

$\mathrm{D}_{\max }=$ maximum point dose.

* The $10 \%$ criterion uses the spinal cord volume 5-6 mm above and below the target volume. Note that these constraints are intended as a summary of practice patterns of experienced spine specialists. However, these constraints are not data driven. They should be utilized with caution and may not be applicable to all clinical scenarios. Evidence-based constraints have been previously published by Sahgal et al..$^{31,32}$ and are cited here as a reference for the interested reader.

data will be essential to further develop this emerging field, we present a summary of the current practice of a group of experienced providers to serve as a foundation. The consensus is that spine SBRT is appropriate in the postoperative setting in patients with limited disease, radioresistant tumors, or for salvage following failure of conventional RT. The target volume should include the entire extent of preoperative tumor and the involved anatomical compartment plus the postoperative residual, in a manner consistent with the consensus contouring guidelines for intact vertebral metastases ${ }^{6}$ but applied to the postoperative setting. This is supported by recent patterns of failure analysis, which found that the location of preoperative epidural disease was more predictive of subsequent failure than the sites of residual epidural disease postoperatively. ${ }^{4}$ Several controversies remain, including circumferential treatment of the epidural space as well as the appropriate margin in patients with soft tissue and paraspinal involvement. While future investigations will be critical in clarifying these concepts, we report many areas of consistency independently adapted by experienced spine oncology specialists driven by their clinical experience and outcomes.

The optimal prescription dose for spine SBRT also remains controversial. Advocates of single-fraction treatment tout potentially higher rates of local control than are achieved with multiple-fraction management, ${ }^{2,19,24}$ perhaps as a result of activation of alternative pathways of cell death, including the sphingomyelinase pathway, ${ }_{10}^{10}$ that manage to overcome the hypoxia-induced resistance paramount to the postoperative setting where tissues are de-vascularized. Proponents of hypofractionated doses criticize the retrospective data for unbalanced patient populations where higher risk patients are by necessity treated more frequently with fractionated treatment plans to respect normal tissue tolerances while covering the larger at-risk target volume than patients with lower risk disease. Accordingly, the higher rates of local control following singlefraction treatment in retrospective series may simply reflect better outcomes in patients with less aggressive disease. ${ }^{14,29}$ In addition, fractionation closes the difference between normal tissue constraints and tumoricidal doses, allowing better coverage of the target volume in the epidural space, the region well established to be at the highest risk of local failure. ${ }^{2,19}$ We summarize reasonable prescription doses and target volume coverage expectations to serve as an important reference to guide practice in the absence of more definitive data. As the body of literature supporting spine SBRT continues to grow, standardization of the reporting of prescription doses will become increasingly important to allow for better understanding of potential dose/response relationships. Specifically, in addition to the prescription dose to the GTV, CTV, and PTV, reporting of dosimetric indices of under-coverage such as the $\mathrm{V}_{95 \%}, \mathrm{~V}_{90 \%}, \mathrm{~V}_{80 \%}$, and $\mathrm{D}_{95 \%}, \mathrm{D}_{90 \%}, \mathrm{D}_{85 \%}$, and $\mathrm{D}_{80 \%}$ is recommended. It may be that upon further standardization we will learn that the variations in prescription doses across studies reflects differences in reporting and institutional standards in plan evaluation rather than in the actual dose received by the target volumes.

Accurate delineation of the spinal cord avoidance structure is paramount to the safe utilization of spine SBRT. This is often complicated in the postoperative patient in whom surgical instrumentation leads to significant hardware artifact. We highlight the importance of precise spinal cord delineation using T2-weighted MRI and/or CT myelography. Furthermore, we report accepted spinal cord tolerances for spine SBRT in the postoperative patient, based on the number of fractions, presence of spinal cord compromise, and exposure to prior radiation. It remains unclear whether these spinal cord constraints should be applied to the true spinal cord or a spinal cord PRV, typically spine cord plus a 1.5-2 mm expansion. Proponents of using the true spinal cord note that this practice allows the greater coverage of the epidural space which is the region at highest risk for recurrence. Those who apply the same dose constraints to a spinal cord PRV retort that this more conservative practice better accounts for errors with set-up, physiological spinal cord motion, and errors in CT/myelogram/MRI fusion. Future investigations will be critical in answering these questions; however, we provide guidelines to promote safe practice in the absence of decisive prospective data.

Finally, treatment planning for SBRT in the postoperative setting is complicated by the presence of surgical 
hardware leading to electron backscatter and photon attenuation. This may not be accurately captured in standard treatment planning algorithms. ${ }^{21,23,26,35} \mathrm{We}$ highlight the importance of employing techniques to calculate dose within a heterogeneous medium and provide a list of algorithms approved by the legacy RTOG.

\section{Conclusions}

This survey of an international group of spine oncology specialists was designed to assist in the development of safe and effective practice recommendations regarding the use of postoperative spine SBRT. There is consensus that postoperative SBRT may be indicated for circumstances of: 1) radioresistant primary histology, 2) disease that is confined to 1-2 vertebral levels, and 3) prior overlapping or adjacent conventional RT. Moreover, the consensus opinion was that postoperative spinal SBRT is contraindicated in circumstances of residual postoperative severe spinal cord compression (Bilsky Grade 3), complete spinal cord injury (ASIA Grade A), and when more than 3 contiguous vertebral levels are involved. For treatment planning, the preoperative MRI and postoperative T1-weighted MRI (with and without gadolinium) should be co-registered and delineation of the cord should be performed using co-registered T1- and/or T2-weighted MRI or a CT myelogram in cases of significant hardware artifact. The GTV is the postoperative residual tumor based on MRI, and the CTV is the postoperative bed defined as the entire extent of preoperative tumor and the involved anatomical compartment plus residual disease. Hardware and scar do not need to be included in CTV. While predominant agreement was reached about treatment planning and definition of organs at risk, future investigation will be critical in better understanding areas of controversy, including whether circumferential treatment of the epidural space is necessary, management of paraspinal extension, and optimal dose fractionation schedules.

\section{References}

1. Ahmed KA, Stauder MC, Miller RC, Bauer HJ, Rose PS, Olivier KR, et al: Stereotactic body radiation therapy in spinal metastases. Int J Radiat Oncol Biol Phys 82:e803-e809, 2012

2. Al-Omair A, Masucci L, Masson-Cote L, Campbell M, Atenafu EG, Parent A, et al: Surgical resection of epidural disease improves local control following postoperative spine stereotactic body radiotherapy. Neuro Oncol 15:1413-1419, 2013

3. Bate BG, Khan NR, Kimball BY, Gabrick K, Weaver J: Stereotactic radiosurgery for spinal metastases with or without separation surgery. J Neurosurg Spine 22:409-415, 2015

4. Chan MW, Thibault I, Atenafu EG, Yu E, John Cho BC, Letourneau D, et al: Patterns of epidural progression following postoperative spine stereotactic body radiotherapy: Implications for clinical target volume delineation. J Neurosurg Spine 24:652-659, 2016

5. Chang EL, Shiu AS, Mendel E, Mathews LA, Mahajan A, Allen PK, et al: Phase I/II study of stereotactic body radiotherapy for spinal metastasis and its pattern of failure. J Neurosurg Spine 7:151-160, 2007

6. Cox BW, Spratt DE, Lovelock M, Bilsky MH, Lis E, Ryu S, et al: International Spine Radiosurgery Consortium consensus guidelines for target volume definition in spinal stereo- tactic radiosurgery. Int J Radiat Oncol Biol Phys 83: 597e605, 2012

7. Epstein-Peterson ZD, Sullivan A, Krishnan M, Chen JT, Ferrone $\mathrm{M}$, Ready J, et al: Postoperative radiation therapy for osseous metastasis: outcomes and predictors of local failure. Pract Radiat Oncol 5:e531-e536, 2015

8. Fehlings MG, Nater A, Tetreault L, Kopjar B, Arnold P, Dekutoski M, et al: Survival and clinical outcomes in surgically treated patients with metastatic epidural spinal cord compression: Results of the prospective multicenter AOSpine study. J Clin Oncol 34:268-276, 2016

9. Fisher CG, Schouten R, Versteeg AL, Boriani S, Varga PP, Rhines LD, et al: Reliability of the Spinal Instability Neoplastic Score (SINS) among radiation oncologists: an assessment of instability secondary to spinal metastases. Radiat Oncol 9:69, 2014

10. Fuks Z, Kolesnick R: Engaging the vascular component of the tumor response. Cancer Cell 8:89-91, 2005

11. Gerszten PC, Burton SA, Ozhasoglu C, Welch WC: Radiosurgery for spinal metastases: clinical experience in 500 cases from a single institution. Spine (Phila Pa 1976) 32:193-199, 2007

12. Gerszten PC, Monaco EA III: Complete percutaneous treatment of vertebral body tumors causing spinal canal compromise using a transpedicular cavitation, cement augmentation, and radiosurgical technique. Neurosurg Focus 27(6):E9, 2009

13. Hartsell WF, Scott CB, Bruner DW, Scarantino CW, Ivker RA, Roach M III, et al: Randomized trial of short- versus long-course radiotherapy for palliation of painful bone metastases. J Natl Cancer Inst 97:798-804, 2005

14. Heron DE, Rajagopalan MS, Stone B, Burton S, Gerszten PC, Dong X, et al: Single-session and multisession CyberKnife radiosurgery for spine metastases-University of Pittsburgh and Georgetown University experience. J Neurosurg Spine 17:11-18, 2012

15. Howell DD, James JL, Hartsell WF, Suntharalingam M, Machtay M, Suh JH, et al: Single-fraction radiotherapy versus multifraction radiotherapy for palliation of painful vertebral bone metastases-equivalent efficacy, less toxicity, more convenient: a subset analysis of Radiation Therapy Oncology Group trial 97-14. Cancer 119:888-896, 2013

16. Jin JY, Chen Q, Jin R, Rock J, Anderson J, Li S, et al: Technical and clinical experience with spine radiosurgery: a new technology for management of localized spine metastases. Technol Cancer Res Treat 6:127-133, 2007

17. Klekamp J, Samii H: Surgical results for spinal metastases. Acta Neurochir (Wien) 140:957-967, 1998

18. Klimo P Jr, Schmidt MH: Surgical management of spinal metastases. Oncologist 9:188-196, 2004

19. Laufer I, Iorgulescu JB, Chapman T, Lis E, Shi W, Zhang Z, et al: Local disease control for spinal metastases following "separation surgery" and adjuvant hypofractionated or highdose single-fraction stereotactic radiosurgery: outcome analysis in 186 patients. J Neurosurg Spine 18:207-214, 2013

20. Lenz M, Freid JR: Metastases to the skeleton, brain and spinal cord from cancer of the breast and the effect of radiotherapy. Ann Surg 93:278-293, 1931

21. Liebross RH, Starkschall G, Wong PF, Horton J, Gokaslan ZL, Komaki R: The effect of titanium stabilization rods on spinal cord radiation dose. Med Dosim 27:21-24, 2002

22. Massicotte E, Foote M, Reddy R, Sahgal A: Minimal access spine surgery (MASS) for decompression and stabilization performed as an out-patient procedure for metastatic spinal tumours followed by spine stereotactic body radiotherapy (SBRT): first report of technique and preliminary outcomes. Technol Cancer Res Treat 11:15-25, 2012

23. Mesbahi A, Nejad FS: Dose attenuation effect of hip pros- 
theses in a 9-MV photon beam: commercial treatment planning system versus Monte Carlo calculations. Radiat Med 25:529-535, 2007

24. Moulding HD, Elder JB, Lis E, Lovelock DM, Zhang Z, Yamada Y, et al: Local disease control after decompressive surgery and adjuvant high-dose single-fraction radiosurgery for spine metastases. J Neurosurg Spine 13:87-93, 2010

25. Patchell RA, Tibbs PA, Regine WF, Payne R, Saris S, Kryscio RJ, et al: Direct decompressive surgical resection in the treatment of spinal cord compression caused by metastatic cancer: a randomised trial. Lancet 366:643-648, 2005

26. Pekmezci M, Dirican B, Yapici B, Yazici M, Alanay A, Gürdalli S: Spinal implants and radiation therapy: the effect of various configurations of titanium implant systems in a single-level vertebral metastasis model. J Bone Joint Surg Am 88:1093-1100, 2006

27. Puvanesarajah V, Lo SL, Aygun N, Liauw JA, Jusué-Torres I, Lina IA, et al: Prognostic factors associated with pain palliation after spine stereotactic body radiation therapy. J Neurosurg Spine 23:620-629, 2015

28. Rades D, Stalpers LJ, Veninga T, Schulte R, Hoskin PJ, Obralic N, et al: Evaluation of five radiation schedules and prognostic factors for metastatic spinal cord compression. J Clin Oncol 23:3366-3375, 2005

29. Redmond KJ, Sahgal A, Foote M, Knisely J, Gerszten PC, Chao ST, et al: Single versus multiple session stereotactic body radiotherapy for spinal metastasis: the risk-benefit ratio. Future Oncol 11:2405-2415, 2015

30. Ryu S, Jin JY, Jin R, Rock J, Ajlouni M, Movsas B, et al: Partial volume tolerance of the spinal cord and complications of single-dose radiosurgery. Cancer 109:628-636, 2007

31. Sahgal A, Ma L, Weinberg V, Gibbs IC, Chao S, Chang UK, et al: Reirradiation human spinal cord tolerance for stereotactic body radiotherapy. Int J Radiat Oncol Biol Phys 82:107-116, 2012

32. Sahgal A, Weinberg V, Ma L, Chang E, Chao S, Muacevic A, et al: Probabilities of radiation myelopathy specific to stereotactic body radiation therapy to guide safe practice. Int $\mathbf{J}$ Radiat Oncol Biol Phys 85:341-347, 2013

33. Sellin JN, Suki D, Harsh V, Elder BD, Fahim DK, McCutcheon IE, et al: Factors affecting survival in 43 consecutive patients after surgery for spinal metastases from thyroid carcinoma. J Neurosurg Spine 23:419-428, 2015

34. Tatsui CE, Stafford RJ, Li J, Sellin JN, Amini B, Rao G, et al: Utilization of laser interstitial thermotherapy guided by real-time thermal MRI as an alternative to separation surgery in the management of spinal metastasis. J Neurosurg Spine 23:400-411, 2015

35. Wang X, Yang JN, Li X, Tailor R, Vassilliev O, Brown P, et al: Effect of spine hardware on small spinal stereotactic radiosurgery dosimetry. Phys Med Biol 58:6733-6747, 2013

36. Wong DA, Fornasier VL, Macnab I: Spinal metastases: the obvious, the occult, and the impostors. Spine (Phila Pa 1976) $15: 1-4,1990$

\section{Disclosures}

Drs. Redmond, Sahgal, and Lo report being members of an oligometastasis research consortium funded through a grant from Elekta AB. Dr. Lo reports having received travel expenses and honoraria for past educational seminars from Varian Medical Systems and Accuray. Dr. Sahgal reports receiving research grants from Elekta $\mathrm{AB}$ and honoraria for past educational seminars from Elekta AB, Medtronic, and Varian Medical Systems. Dr. Chang has received an Elekta users group meeting honorarium. Dr. Barani has research grants from BrainLab Inc and the International Gamma Knife Research Foundation (IGKRF). Dr. Yamada reports a consultant relationship with Varian Medical Systems and being on the medical advisory board of the Chordoma Foundation. Dr. Chao reports receiving an honorarium from Varian.

\section{Author Contributions}

Conception and design: Redmond, Sahgal. Acquisition of data: Redmond, Lo, Sahgal. Analysis and interpretation of data: Redmond, Lo, Soltys, Yamada, Barani, Brown, Gerszten, Chao, Amdur, De Salles, Guckenberger, Teh, Sheehan, Kersh, Fehlings, Sohn, Chang, Ryu, Gibbs, Sahgal. Drafting the article: Redmond, Lo, Soltys, Chang, Gerszten, Sahgal. Critically revising the article: Redmond, Lo, Soltys, Yamada, Barani, Brown, Chang, Chao, Amdur, De Salles, Guckenberger, Teh, Sheehan, Kersh, Fehlings, Sohn, Chang, Ryu, Gibbs, Sahgal. Reviewed submitted version of manuscript: all authors. Approved the final version of the manuscript on behalf of all authors: Redmond. Statistical analysis: Redmond. Administrative/technical/material support: Redmond. Study supervision: Redmond, Lo, Sahgal. Survey participants: all authors.

\section{Supplemental Information \\ Online-Only Content}

Supplemental material is available with the online version of the article.

Appendix. https://thejns.org/doi/suppl/10.3171/2016.8. SPINE16121.

\section{Current Affiliations}

Dr. Lo: University of Washington School of Medicine, Seattle, WA. Dr. Brown: Mayo Clinic, Rochester, MN.

\section{Correspondence}

Kristin J. Redmond, Department of Radiation Oncology and Molecular Radiation Sciences, The John Hopkins University, 401 North Broadway, Ste. 1440, Baltimore, MD 21231. email: kjanson3@jhmi.edu. 